\title{
Improving IA-RWA Algorithms in Translucent Networks by Regenerator Allocation
}

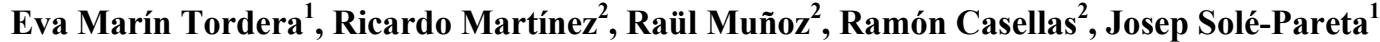 \\ ${ }^{1}$ Technical University of Catalonia (UPC), Jordi Girona, 1-3. 08034 Barcelona, Catalonia, Spain \\ \{eva,pareta\}@ac.upc.edu \\ ${ }^{2}$ Centre Tecnolòic de Telecomunicacions de Catalunya, Av.Canal Olímpic s/n, 08860 Castelldefels, Spain \\ \{ricardo.martinez, raul.munoz, ramon.casellas\}@cttc.es
}

\begin{abstract}
In this paper we present the impact of considering regenerator allocation when selecting routes and wavelengths in translucent networks. In the regular operation of translucent networks, i.e. with dynamic traffic, we assume that a certain number of $3 \mathrm{R}$ regenerators are installed in some nodes of the network. These regenerators break the optical transparency of the lightpaths, but allow establishing the optical connections with the required optical signal quality. We show the performance improvement of the MINCOD-Q IA-RWA algorithm when an efficient regenerator allocation policy is employed (optical regeneration is only performed when the signal quality goes bellow a pre-established threshold). Under this policy, the (extended) MINCOD-Q algorithm performs slightly better in terms of blocking probability, but and most important, this figure is obtained with a significant reduction of the number of $3 \mathrm{R}$ regenerators installed in the network.
\end{abstract}

\section{INTRODUCTION}

The evolution of optical networks is moving from an opaque architecture, in which the optical signal is converted to the electronic domain and back to the optical domain at each node, towards an all-optical (transparent) architecture. The main motivation behind this trend is the cost savings achieved by the elimination of a significant amount of electronic equipment. Transparent optical networks do not convert the signal to the electrical domain in the intermediate nodes, but rather keep the signal within the optical domain from the source node to the destination node. However, full transparency is not always achievable in long distance networks due to the degradation an optical signal accumulates whilst propagating towards the receiver. In translucent optical networks, regenerators are thus employed when the quality of the optical signal falls below the level required for an acceptably correct detection. Since the regenerator breaks up the optical continuity, the optical network becomes a translucent optical network. Regeneration is essential to re-amplify, re-shape and re-time the optical signal (i.e., 3R or simply regeneration). The incorporation of the physical impairments that degrade the optical signal in the Routing and Wavelength Assignment (RWA) for transparent and translucent optical networks, known as Impairment Aware RWA or IA-RWA, has recently received some attention from the research communities. A survey of IA-RWA algorithms in optical networks can be found in [1].

When designing optical networks, physical impairments have to be taken into account in order to know how many, and in which nodes, regenerators are needed. This procedure is known as regenerator placement. To this end, static traffic matrixes and static IA-RWA algorithms are used. On the other hand, during the regular operation (i.e., dynamic traffic) the on-line IA-RWA algorithm decides which regenerators (already installed) are used or activated. This problem is known as regenerator allocation.

This paper deals with the regenerator allocation in translucent networks. We present the improvement when using regenerator allocation in an IA-RWA algorithm for dynamic traffic in terms of both connection blocking and regenerator resource consumption when regenerators are used efficiently rather than systematically. We show that when using regenerator allocation efficiently, the IA-RWA algorithms behave better (that is they require less regenerator per unit time), which in turn leads to reduce the consumption of energy.

\section{IA-RWA ALGORITHMS WITH REGENERATOR PLACEMENT AND ALLOCATION IN TRANSLUCENT NETWORKS}

Two key problems are typically addressed in translucent optical networks, namely, the regenerator placement and the regenerator allocation at the time of establishing connections. The former relies on how planning the most cost-efficient network when placing regenerators. This results a complex optimization problem which is solved by off-line IA-RWA algorithms during the design network stage. To face such complexity, heuristics such as the Nodal Degree First algorithm, the Centered Node First scheme, and the Traffic Load and Signal Quality Predictions strategies are employed [2], [3].

Once the regenerator resources are placed, the second problem involves the routing of the connection requests, in order not only to satisfy a set of restrictions such as adequate optical signal quality and wavelength continuity (WCC), but also to attain an efficient utilization of the network resources (i.e., wavelength channels and $3 \mathrm{R}$ regenerators). To this end, several IA-RWA algorithms have been proposed in the literature, classified 
into two categories depending on the employed constraint to address the optical signal quality: algorithms which do not exceed a maximum transparent distance, otherwise regeneration is needed; and IA-RWA algorithms that consider analytical expressions for particular physical impairments such as the optical signal noise ratio (OSNR), the bit error rate (BER), the Q factor, etc. In the first category, [4] proposes the 2 Dimensional (2D) algorithm which is an-online IA-RWA mechanism which computes the shortest path not exceeding the regeneration distance limits. For the second category, proposes an IA-RWA algorithm considers the OSNR, the polarization mode dispersion and the chromatic dispersion as optical constraints for regenerator allocation. In [5] the authors presented an on-line OSNR-based IA-RWA algorithm, deployed in a GMPLS network, which considers the received OSNR level as the optical signal performance parameter, dynamically allocating available regenerators if this value is below a given OSNR threshold. In the present work, however, the IA-RWA algorithm relies on the Personick's Q-factor [6] as the optical signal performance parameter.

\subsection{Personick's Q-Factor}

Given the network topology designed during the dimensioning phase, the connection path assigned by the routing operation, the wavelengths assigned to the connection at each traversed line system, and the placement of regenerators, it is possible to ascertain whether the final BER at the receiver is below a specified threshold. To this end, an effective method for estimating the optical signal degradation along a path is required. This can be tackled by observing that the BER threshold can be translated into a threshold value of the so-called Personick $Q$-factor [6]. The $Q$ factor can be evaluated as a function of the transmission-system parameters and the transmission impairments [7]. Without any error correction mechanism on the digital signal at the receiver, a quality factor $Q=16.9 \mathrm{~dB}$ corresponds to a BER of approximately $1 \times 10^{-12}$. Typically, the requirements for the minimum value of $Q$ of a signal at the receiver are about $17 \mathrm{~dB}$ without error correction, and $12 \mathrm{~dB}$ in case of error correction. The expression used for evaluating the $Q$ factor at the receiver endpoint of a transparent path (or sub-path) is given by (1).

$$
Q_{\text {end }}=a_{0}+a_{1} O_{S N R_{\text {end }}}+a_{2} N_{S P A N}+a_{3}\left(P_{0} N_{S P A N}\right)^{B}
$$

According to (1) the quality factor depends on both linear and non-linear effects. The $O S N R_{\text {end }}$ factor is the optical signal to noise ratio expressed in $\mathrm{dB}$ at the receiver. The terms $a_{2} N_{S P A N}$ and $a_{3}\left(P_{0} N_{S P A N}\right)^{\mathrm{B}}$ take into account the non-linear effects, considering all the amplifiers along a path (booster, pre- and line amplifiers). $N_{S P A N}$ is the number of spans forming the transparent path. where a span is the segment of a link between two amplifiers. $P_{0}[\mathrm{dBm}]$ is the power level at the signal transmission (typically $3 \mathrm{dBm}$ ). The coefficients $a_{0}, a_{1}, a_{2}$, $a_{3}$, and $B$ depend on the type of used line systems, and should be tuned by an on-field measurement campaign.[7]

\subsection{MINCOD-Q Algorithm}

In this work, we consider the so-called Minimum Coincidence and Distance according to $Q$ factor (MINCOD-Q) as the IA-RWA algorithm, based on the Minimum Coincidence and Distance (MINCOD) routing algorithm [8]. MINCOD-Q uses a set of $K$ pre-computed routes, from a source node to a destination node, having the minimum distance in terms of $\mathrm{Km}$ and the minimum number of shared links. The $Q$ factor is computed according to the expression (1), and is used as the performance indicator to decide whether a lightpath or the set of transparent sub-paths (i.e. transparent segments) satisfy a given $Q$-threshold constraint. For each sub-path, the selected wavelength channel is the first one available in each link forming the sub-paths. Recall, that a $3 \mathrm{R}$ regenerator also allows wavelength conversion capability

In short, the MINCOD-Q algorithm selects amongst the $K$ pre-computed routes a path and the wavelength channels that allow fulfilling both the WCC and the optical signal quality restriction in each sub-path, always using pre-planned placed regenerators if included in the path.

\subsubsection{GMPLS Control Plane Extensions for Translucent Networks}

The utilization of a distributed control plane is a key element to introduce intelligence and automatic mechanisms (routing and signaling) in optical networks. The most deployed control plane architecture is Generalized Multi Protocol Label Switching (GMPLS), a protocol suite defining the signaling, routing and link management protocols and functions to support the automatic provisioning of end-to-end traffic engineering connections.

Standard GMPLS protocols (signaling and routing) are well-defined for opaque networks. Indeed, in such infrastructures the WCC and the end-to-end optical signal quality are not issues. In the context of transparent networks, however, some routing extensions are required to both disseminate physical layer attributes (e.g., OSNR) and to flood resource information at wavelength granularity in order to route lightpaths with acceptable optical signal level at the receiver while addressing the WCC. Besides these extensions, the potential utilization of sparse regenerators in translucent networks does require additional routing protocol enhancements in order to convey the availability of the regenerators. That is, the IA-RWA algorithm needs to have updated information not only about the network topology and the available wavelengths on each link, but also about the network nodes having unused regenerators. In [5], the authors presented and validated enhancements to both GMPLS- 
based routing and signaling protocols to provide the required information to execute IA-RWA algorithms within translucent networks.

\section{IMPROVING MINCOD-Q ALGORITHM BY REGENERATOR ALLOCATION}

In the MINCOD-Q algorithm, a connection is set up allocating an available regenerator in any of the intermediate translucent nodes along the route where they are installed. In other words, if we assume that only some nodes of the network are equipped with 3R regenerators, MINCOD-Q will consume as many regenerators as nodes with free regenerators finds in the nodes forming the path. Note that we assume that a regenerator placement algorithm was used to place the regenerators according to a static traffic matrix. Consequently, MINCOD-Q does not achieve an efficient utilization of the network resources (3R regenerators), since it is very likely that some regenerators will be allocated in a path without actually being needed to fulfil either the WCC or the adequate optical signal quality requirements. Thereby, in this work, we enhance the MINCOD-Q routing decision in order to use the regenerators installed in the network only if they are necessary. As a result, for establishing a connection, the enhanced MINCOD-Q algorithm computes the path without allocating regenerators up to the first node wherein the $Q$ factor level is below a predetermined $Q$ threshold. There the algorithm goes back and looks for a regenerator in the closer downstream node where it can be found, and at the same time the $Q$ factor level of the signal is higher than the threshold. Once such a node is found, the regenerator is allocated to that connection. This causes that the provisional path of that connection will be at least divided in two transparent sub-paths/segments. We call this new algorithm as MINCOD-Q-REG algorithm.

\section{PERFORMANCE EVALUATION}

In order to evaluate the proposed MINCOD-Q REG algorithm we have carried out a set of simulations under the Pan European network with 28 nodes and 41 links, where all links were equipped with systems of 40 wavelengths. The number of 40 wavelengths systems installed in parallel on each link was calculated by a planning procedure. On the basis of a uniform static traffic matrix, the planning tool gave us 129 regenerators distributed in 19 of the nodes, and 56 systems distributed in the 41 links. More detailed information about the planning stage can be found in [7].

We carried out the simulations under dynamic traffic conditions, from 0.1 to 1 Erlang. For all the numerical results, the I threshold is set to $15 \mathrm{~dB}$. These results are depicted in Fig. 1. Figure 1a and 1b show the percentage of blocked connections and the average number of regenerators used versus the traffic load, considering 129 regenerators for both 2-MINCOD-Q and 2-MINCOD-Q REG algorithms, respectively. In both algorithms, the number of pre-computed path is 2 ( $K$ parameter), and they are computed according the MINCOD algorithm [8]. We observe that the 2-MINCOD-Q REG algorithm achieves a slightly reduction of blocked connection and at the same time a significant reduction of regenerators in use.

Figure $1 \mathrm{c}$ and $1 \mathrm{~d}$ depicts the same results but when the number of installed regenerators in the network is 14 ,

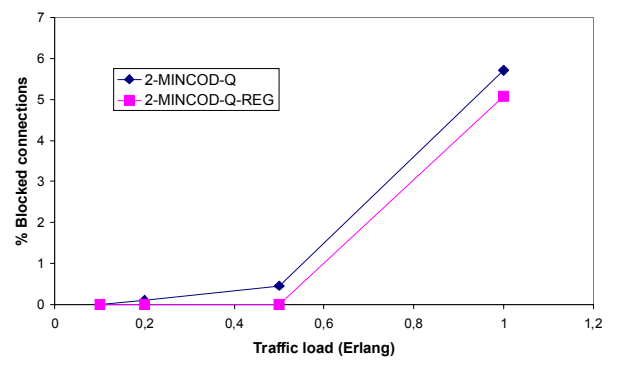

a) Blocked connections for 129 installed regenerators

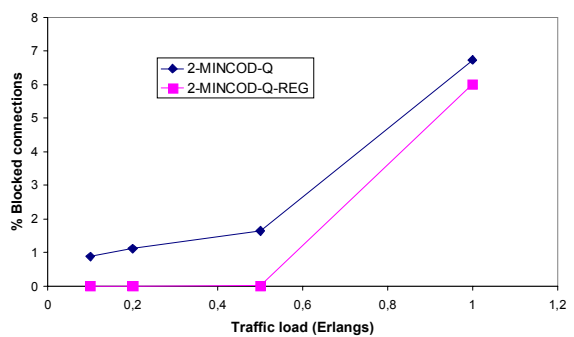

c) Blocked connections for 14 installed regenerators

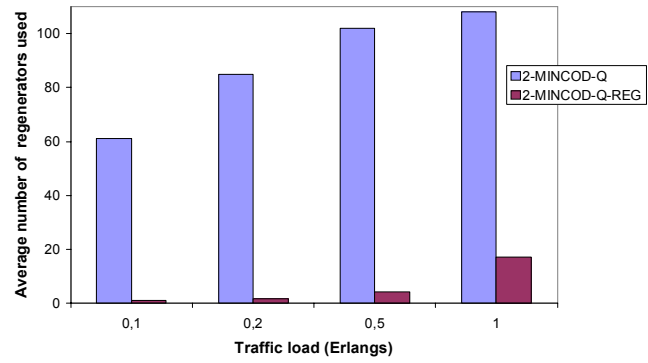

b) Average regenerators used for 129 regenerators

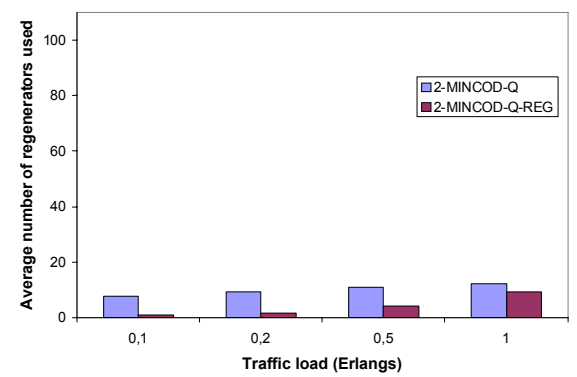

d) Average regenerators used for 14 regenerators.

Figure 1. Percentage of blocked connections and Average Number of regenerators used versus traffic load. 
in 14 of the 28 nodes. In general, the performance of the 2-MINCOD-Q REG algorithm is less affected by the regenerator reduction. Only when the traffic load is high (1 Erlang) the increase in blocking probability is noticeable Fig. 2 shows the comparison between the results of the 2-MINCOD-Q algorithm with 129 installed regenerators and the results of 2-MINCOD-Q REG with only 14 installed regenerators. We observe in Fig. 2 that 2-MINCOD-Q REG performs close to 2-MINCOD-Q in terms of blocking probability with only 11\% (14) of installed regenerators. On the other hand, it is worth noting that MINCOD-Q algorithm is highly dependable of the number of placed or regenerators. Consequently, a variation of such a number causes an appreciable impact on the blocked connections. Moreover the benefits in terms of CAPEX and also regenerator consumption of the 2-MINCOD-Q REG are even more important. With only 10 regenerators used in mean (see Fig. 2a) we achieve a $0 \%$ of blocked connections from 0.1 to 0.5 Erlangs.

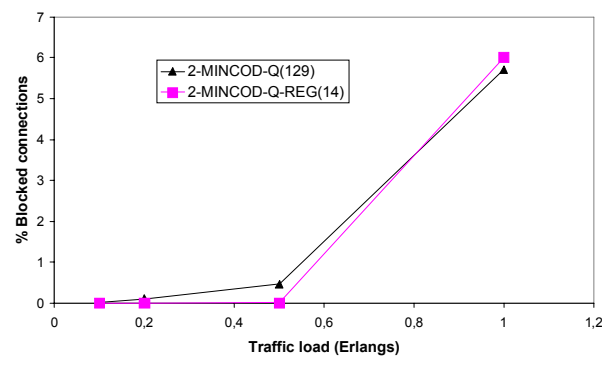

a) Blocked connections versus traffic load

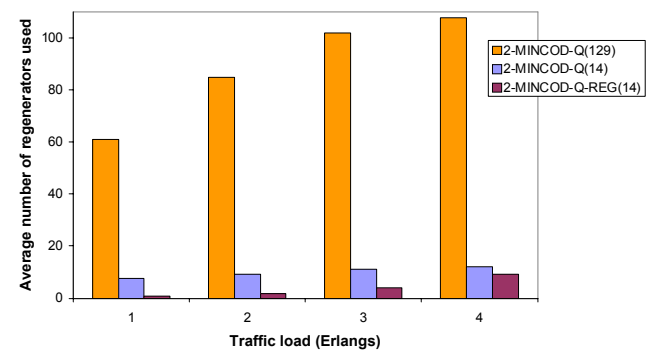

b) Average number of regenerators used

Figure 2. Comparison of 2-MINCOD-Q verus 2-MINCOD-Q REG.

\section{CONCLUSIONS}

In this paper we have presented how the efficient regenerator allocation can improve the performance of the IARWA algorithms. We have compared two versions of a IA-RWA algorithm, namely pure MINCOD-Q where an efficient use of 3R regenerator is not considered and 2-MINCOD-Q REG where a regenerator allocation strategy is applied. The improved IA-RWA algorithm (2-MINCOD-Q REG) allocates the regenerators at the nodes where they are available only when the signal quality goes below a pre-established threshold. This allows attaining improvements in terms of not only blocking probability, but also and significant in terms of resource utilization (regenerators), of specific impact in CAPEX and energy consumption aspects. The main outcome of this work is that reaching practically the same blocking rate, the improved version of our algorithm reduces the number regenerators to be installed in the network up to $90 \%$, which implies a significant CAPEX reduction.

\section{ACKNOWLEDGEMENTS}

This work was partially funded by the MICINN (Spanish Ministry of Science and Innovation) under the RESPLANDOR project (TEC2006-12910/TCM), and by the European Commission under the BONE (Building the Future Optical Network in Europe) Network of Excellence of the 7th ICT Framework Programme.

\section{REFERENCES}

[1] S. Azodolmolky, M. Klinkowski, E. Marin, D. Careglio, J. Sole Pareta, I. Tomkos., “A survey on physical layer impairments aware routing and wavelength assignment algorithms in optical networks", Computer Networks (Elsevier) (ISSN: 1389-1286), Accepted for publication.

[2] X. Yang, and B. Ramamurthy, "Dynamic routing in translucent WDM optical networks the intra-domain case”, Journal of Lightwave Technology, vol. 23, no. 3, pp. 955-71, Mar. 2005.

[3] X. Yang and B. Ramamurthy, "Sparse regeneration in translucent wavelength-routed networks: Architecture, network design and wavelength routing," Photonic Network Com.., vol. 10, no. 1, pp. 39-53, Jan. 2005.

[4] G. Shen et. al., "Sparse placement of electronic switching node for low blocking in translucent optical networks", OSA J. Opt. Networking, vol. 1, no. 12, pp. 424-441, Dec. 2002.

[5] R. Martínez et. al., "Experimental GMPLS routing for dynamic provisioning in translucent wavelength switched optical networks", in Proc. OFC/NFOEC 2009, San Diego, USA, March 2009.

[6] S.D. Personick, "Receiver Design for Digital Fiber Optic Communication Systems, I", Bell Syst. Tech. J, vol. 52, no. 6, Jul.-Aug., 1973, pp. 843-874.

[7] M. Yannuzzi et. al., "Performance of translucent optical networks under dynamic traffic and uncertain physical-layer information", in Proc. ONDM 2009.

[8] E. Marín-Tordera et al., "MINCOD-MTD: A RWA algorithm in semi-transparent optical networks", in Proc. ECOC 2007. 